\title{
Additive properties of a pair of sequences
}

\author{
by \\ Fabricio Benevides (Memphis, TN), \\ Jonathan Hulgan (Memphis, TN), \\ Nathan Lemons (Budapest), Cory Palmer (Budapest), \\ Ago-Erik Riet (Memphis, TN), and \\ Jeffrey PAul Wheeler (Pittsburgh, PA)
}

1. Introduction. For a given set $A \subset \mathbb{N}_{0}$ of non-negative integers, here and throughout the paper, the counting function $A(n)$ is defined as the number of elements of $A$ not exceeding $n$, i.e., $A(n)=|A \cap\{0,1, \ldots, n\}|$. Consider the following functions:

$$
\begin{aligned}
r(A, n) & =\left|\left\{\left(a_{1}, a_{2}\right) \in A \times A: a_{1}+a_{2}=n\right\}\right|, \\
r_{1}(A, n) & =\mid\left\{\left(a_{1}, a_{2}\right) \in A \times A: a_{1}+a_{2}=n \text { and } a_{1} \leq a_{2}\right\} \mid, \\
r_{2}(A, n) & =\mid\left\{\left(a_{1}, a_{2}\right) \in A \times A: a_{1}+a_{2}=n \text { and } a_{1}<a_{2}\right\} \mid .
\end{aligned}
$$

A well-studied problem concerning these functions is to determine necessary and sufficient conditions on $A$ for their (eventual) monotonicity. Here and throughout the paper, monotonicity refers to monotonicity in $n$. In other words, for what sets $A$ can we find an $n_{0}$ such that $r(A, n+1) \geq r(A, n)$ for all $n>n_{0}$ ? Although the three functions look similar, and in fact $\mid r(A, n)-$ $2 r_{2}(A, n) \mid \leq 1$ and $\left|r_{1}(A, n)-r_{2}(A, n)\right| \leq 1$, the (partial) answers to these questions may be quite different.

Erdős, Sárközy and Sós [3] proved that $r(A, n)$ is eventually increasing if and only if $A$ contains all the positive integers from a certain point on. On the other hand, they obtained only a partial answer for $r_{1}$ and $r_{2}$. In particular, they proved that if

$$
\lim _{n \rightarrow \infty} \frac{n-A(n)}{\log n}=\infty
$$

then $r_{1}(A, n)$ is not eventually increasing. (This result was also obtained independently by Balasubramanian [1].)

2000 Mathematics Subject Classification: Primary 11B34; Secondary 11P99.

Key words and phrases: Sidon set, sum set, representation function, monotonicity. 
Also, for $r_{2}(A, n)$ they proved that if

$$
A(n)=o\left(\frac{n}{\log n}\right)
$$

then $r_{2}(A, n)$ cannot be increasing from a certain point on.

Motivated by these results, Sárközy asked the following question in his valuable paper [8] on unsolved problems in number theory (see Problem 4 in $[8])$.

Problem 1. If $A, B$ are given infinite sets of non-negative integers, what can one say about the monotonicity of the number of solutions of the equation

$$
a+b=n, \quad a \in A, b \in B ?
$$

We can naturally rephrase this question by defining the following function.

Definition 2. The representation function for two sets $A, B \subset \mathbb{N}_{0}$ is

$$
r(A, B, n)=|\{(a, b) \in A \times B: a+b=n\}| .
$$

The main goal of the present paper is to give some sufficient conditions on $A, B$ for the monotonicity of this function. This new representation function acts surprisingly different from the preceding functions. Our main result is as follows.

Theorem 3. For all $0 \leq \alpha, \beta<1,1 / 2<c_{1}, c_{2} \leq 1$, there exist sets $A, B \subset \mathbb{N}_{0}$ such that $r(A, B, n)$ is increasing in $n$, and

$$
\limsup _{n \rightarrow \infty} \frac{A(n)}{n^{c_{1}}}=\alpha, \quad \limsup _{n \rightarrow \infty} \frac{B(n)}{n^{c_{2}}}=\beta .
$$

In the next sections we develop tools to approach Theorem 3 and prove some related results. Then we will return to the proof of Theorem 3 .

2. Co-Sidon sets. Before proving Theorem 3, we introduce a generalized notion of Sidon sets and study some of its properties. Recall that a set $A \subset \mathbb{N}_{0}$ is called Sidon if $r_{1}(A, n) \leq 1$ for all $n \in \mathbb{N}$, i.e., the sums of unordered pairs of elements of $A$ are all distinct. We remark that it is possible to extend the notion of a Sidon set to a pair of sets in different ways. In this paper, we consider the following generalization.

Definition 4. Two sets $A, B \subset \mathbb{N}_{0}$ are called co-Sidon if $r(A, B, n) \leq 1$ for all $n \in \mathbb{N}_{0}$, i.e., the sums $a+b$ are distinct for all $(a, b) \in A \times B$.

Note that if $A, B$ are co-Sidon then $|A \cap B| \leq 1$.

For sets $A$ and $B$ of integers we denote their sum set by $A+B=\{a+b$ : $a \in A, b \in B\}$. For simplicity, if the set $B$ is a single element $b$ we denote their sum set by $A+b=A+B$. 
When $A, B$ are finite sets, we prove a simple but sharp result about $|A|,|B|$.

Theorem 5. If $A, B \subset\{0,1, \ldots, n\}$ are co-Sidon, then

$$
\min \{|A|,|B|\} \leq\lfloor\sqrt{2 n}\rfloor .
$$

Furthermore, equality can be obtained for infinitely many values of $n$.

Proof. Since $A$ and $B$ are finite (and co-Sidon) we have $|A+B|=|A||B|$. Without loss of generality assume $|A| \leq|B|$. Then $|A|^{2} \leq|A+B|$.

Clearly, for an element $c \in A+B$ we have $0 \leq c \leq 2 n$. However, either 0 or $2 n$ is not an element of $A+B$, otherwise we would have $0, n \in A \cap B$ and there would be two distinct solutions to $a+b=n$ with $a \in A$ and $b \in B$. Thus, $|A+B| \leq 2 n$, which yields $|A| \leq\lfloor\sqrt{2 n}\rfloor$, and the upper bound is established.

To see that the upper bound is best possible for infinitely many $n$, consider the following construction for $A$ and $B$. Let $m \in \mathbb{N}$ be fixed and define

$$
\begin{aligned}
& A:=\{0, m, 2 m, \ldots,(2 m-1) m\}, \\
& B:=\left\{0,1,2, \ldots, m-1,2 m^{2}, 2 m^{2}+1,2 m^{2}+2, \ldots, 2 m^{2}+m-1\right\} .
\end{aligned}
$$

Note that $|A|=|B|=2 m$ and $A+B=\left\{0,1, \ldots, 4 m^{2}-1\right\}$. Therefore $A$ and $B$ are co-Sidon. As $A, B \subseteq\left\{0,1, \ldots, 2 m^{2}+m-1\right\}$, we can take $n=2 m^{2}+m-1$. This gives

$$
2 m=\sqrt{4 m^{2}} \leq \sqrt{4 m^{2}+2 m-2}=\sqrt{2 n}<\sqrt{4 m^{2}+4 m+1}=2 m+1 .
$$

Hence $\min \{|A|,|B|\}=2 m=\lfloor\sqrt{2 n}\rfloor$. As the choice of $m$ was arbitrary, there are infinitely many $n$ for which we can reach the upper bound in the statement of the theorem.

The above result can be compared with the following theorem of Erdös and Turán [4] on finite Sidon sets.

THEOREM 6. There is an absolute positive constant $c$ such that if $n \in \mathbb{N}$ and $A \subset\{1, \ldots, n\}$ is a Sidon set, then $|A|<n^{1 / 2}+c n^{1 / 4}$.

On the other hand, the best known constructions give Sidon sets of size $n^{1 / 2}$ for infinitely many $n$ (see e.g. [5, 7] for details). The reduction of this gap is a well-known hard problem.

We now consider the case where $A, B$ are infinite co-Sidon. Defining $A_{n}=A \cap\{0,1, \ldots, n\}$ and $B_{n}=B \cap\{0,1, \ldots, n\}$, we see that $A_{n}, B_{n}$ are co-Sidon. So, by Theorem 5 , for any $n$ we have

$$
\min \{A(n), B(n)\} / \sqrt{n}=\min \left\{\left|A_{n}\right|,\left|B_{n}\right|\right\} / \sqrt{n} \leq\lfloor\sqrt{2 n}\rfloor / \sqrt{n} \leq \sqrt{2} .
$$

A simple example shows that we can come close to achieving this bound.

Construction 7 . Let $A$ be the set of integers which can be written in the form $\sum_{i=0}^{k} \alpha_{i} 2^{2 i}$ where $\alpha_{i} \in\{0,1\}$ and $k \in \mathbb{N}$. Let $B$ be the set of 
integers which can be written in the form $\sum_{i=0}^{k} \alpha_{i} 2^{2 i+1}$ where $\alpha_{i} \in\{0,1\}$ and $k \in \mathbb{N}$. It is clear that $A$ and $B$ are co-Sidon and $A+B=\mathbb{N}_{0}$. It can easily be verified that

$$
\begin{array}{ll}
\liminf _{n \rightarrow \infty} \frac{A(n)}{\sqrt{n}}=1, & \limsup _{n \rightarrow \infty} \frac{A(n)}{\sqrt{n}}=\sqrt{3}, \\
\liminf _{n \rightarrow \infty} \frac{B(n)}{\sqrt{n}}=\frac{\sqrt{2}}{2}, & \limsup _{n \rightarrow \infty} \frac{B(n)}{\sqrt{n}}=\frac{\sqrt{6}}{2} .
\end{array}
$$

Thus,

$$
\liminf _{n \rightarrow \infty} \frac{\min \{A(n), B(n)\}}{\sqrt{n}}=\frac{\sqrt{2}}{2} .
$$

Comparing this with the following result of Erdős (see $[9,5]$ ), we conclude that infinite Sidon sets and infinite co-Sidon sets also behave differently. In general, we have more freedom when working with co-Sidon sets.

THEOREM 8. There is an absolute, positive constant $c$ such that for any infinite Sidon set $A \subset \mathbb{N}$ we have

$$
\liminf _{n \rightarrow \infty} \frac{A(n)}{\sqrt{n / \log n}}<c .
$$

The following theorem of Krückeberg [6] for infinite Sidon sets is also worth mentioning.

TheOREM 9. There is a Sidon set $A \subset \mathbb{N}$ such that

$$
\limsup _{n \rightarrow \infty} \frac{A(n)}{\sqrt{n}} \geq \frac{\sqrt{2}}{2} \text {. }
$$

The following definition will be useful for us.

Definition 10. We call sets $A, B \subset \mathbb{N}_{0}$ perfect if the sum set $A+B$ is an interval (possibly unbounded) of consecutive integers.

The next proposition will be helpful in building new perfect co-Sidon sets from other co-Sidon sets.

Proposition 11. Let $A, B \subset \mathbb{N}_{0}$ be finite perfect co-Sidon sets. Let $c=\max (A)+\max (B)-\min (A)-\min (B)+1$. Then for any $k \in \mathbb{N}_{0}$, the sets $A$ and $C=\bigcup_{i=0}^{k}(B+i c)$ are perfect co-Sidon.

Proof. Let $r=\min (A)+\min (B)$. By assumption, $A+B=\{r, r+1$, $\ldots, c+r-1\}$. For each $i$, the sets $A$ and $B+i c$ are co-Sidon. Furthermore, 
the sets

$$
\begin{aligned}
A+(B+c) & =\{c+r, c+r+1, \ldots, 2 c+r-1\} \\
A+(B+2 c) & =\{2 c+r, 2 c+r+1, \ldots, 3 c+r-1\} \\
& \vdots \\
A+(B+k c) & =\{k c+r, k c+r+1, \ldots,(k+1) c+r-1\}
\end{aligned}
$$

are all pairwise disjoint consecutive intervals. Therefore $A$ and $\bigcup_{i=0}^{k}(B+i c)$ are perfect co-Sidon with sum set $\{r, r+1, \ldots,(k+1) c+r-1\}$.

Clearly, the proposition also holds for $C=\bigcup_{i=0}^{\infty}(B+i c)$.

Next we characterize all infinite perfect co-Sidon sets $A, B \subset \mathbb{N}_{0}$ using the mixed-radix representation. Note that both the co-Sidon and perfect properties are invariant under translation of each of the sets (i.e. addition or subtraction of a constant), so without loss of generality we may assume $0 \in A \cap B$.

TheOREM 12. Let $A, B \subset \mathbb{N}_{0}$ be infinite, such that $0 \in A \cap B$. Then $A, B$ are perfect co-Sidon if and only if there exists an infinite sequence of integers $\left(k_{i}\right)_{i=1}^{\infty}$ such that $k_{i} \geq 2$ for all $i$, and (up to an exchange of $A$ and $B$ )

$$
\begin{aligned}
& A=\left\{\sum_{i=1}^{\infty} k_{1} k_{2} \cdots k_{2 i-2} a_{2 i-1}: \forall j, 0 \leq a_{2 j-1}<k_{2 j-1},\right. \\
& \left.\quad \text { finitely many } a_{2 i-1} \text { non-zero }\right\}, \\
& B=\left\{\sum_{i=1}^{\infty} k_{1} k_{2} \cdots k_{2 i-1} a_{2 i}: \forall j, 0 \leq a_{2 j}<k_{2 j},\right.
\end{aligned}
$$

finitely many $a_{2 i}$ non-zero $\}$.

Proof. A sum of the form $\sum_{i=1}^{\infty} k_{1} k_{2} \cdots k_{i-1} a_{i}$, where $0 \leq a_{j}<k_{j}$ and only finitely many $a_{i}$ are non-zero, is precisely the so-called mixed-radix representation with bases $\left(k_{1}, k_{2}, \ldots\right)$. Thus the base $r$ representation is the special case where $k_{i}=r$ for all $i$. For any sequence $\left(k_{i}\right)_{i=1}^{\infty}$ of integers with $k_{i} \geq 2$, every non-negative integer is uniquely representable with bases $\left(k_{i}\right)$.

Let $\left(k_{i}\right)_{i=1}^{\infty}$ be a sequence of integers such that $k_{i} \geq 2$ for all $i$. Suppose $A$ and $B$ are of the form determined by the bases $k_{i}$ as above. As every non-negative integer is uniquely representable with bases $\left(k_{i}\right), A$ and $B$ are co-Sidon. Also observe that

$$
A+B=\left\{\sum_{i=1}^{\infty} k_{1} k_{2} \cdots k_{i-1} a_{i}: \forall j, 0 \leq a_{j}<k_{j} \text {, finitely many } a_{i} \text { non-zero }\right\} \text {. }
$$

Thus $A+B=\mathbb{N}_{0}$ and therefore $A$ and $B$ are perfect. 
Now assume that $A, B$ are perfect co-Sidon. Unless $A=B=\{0\}$, we can assume without loss of generality that $1 \in A$. To show that $A, B$ are of the required form, we need to construct a sequence of base elements $\left(k_{i}\right)_{i \in \mathbb{N}}$ that represents $A$ and $B$ as in the statement of the theorem.

Our construction of the integers $k_{i}$ is recursive. Let $k_{0}=1$. For $t \geq 1$ define $c_{t}=k_{t-1} k_{t-2} \cdots k_{0}$ and let

$$
k_{t}= \begin{cases}\max \left\{a:\left\{c_{t}, 2 c_{t}, \ldots,(a-1) c_{t}\right\} \subset A\right\} & \text { if } t \text { is odd } \\ \max \left\{b:\left\{c_{t}, 2 c_{t}, \ldots,(b-1) c_{t}\right\} \subset B\right\} & \text { if } t \text { is even. }\end{cases}
$$

Note that $k_{t}<\infty$ for all $t>0$. Otherwise, one of $A$ or $B$ contains an infinite arithmetic progression, whose consecutive terms differ by $c_{t}$. But as they are co-Sidon, this implies that the other set is finite (in fact of cardinality at most $c_{t}$ ), a contradiction.

Now define two families of sets. Let $A_{0}=B_{0}=\{0\}$ and, for each $t \geq 1$,

$$
\begin{aligned}
& A_{t}=\left\{\sum_{i=1}^{t} k_{1} k_{2} \cdots k_{i-1} a_{i}: \forall j, 0 \leq a_{j}<k_{j} \text { and } a_{2 j}=0\right\}, \\
& B_{t}=\left\{\sum_{i=1}^{t} k_{1} k_{2} \cdots k_{i-1} b_{i}: \forall j, 0 \leq b_{j}<k_{j} \text { and } b_{2 j-1}=0\right\} .
\end{aligned}
$$

Note that for all $j, A_{2 j}=A_{2 j-1}$ and $B_{2 j-1}=B_{2 j-2}$. Let $A^{*}=\bigcup_{i=0}^{\infty} A_{t}$ and $B^{*}=\bigcup_{i=0}^{\infty} B_{t}$. It only remains to prove that $A=A^{*}$ and $B=B^{*}$. We will use the following claim.

Claim 13. For all $t \geq 0$,

$$
A \cap\left\{0,1, \ldots, k_{1} \cdots k_{t}-1\right\}=A_{t}, \quad B \cap\left\{0,1, \ldots, k_{1} \cdots k_{t}-1\right\}=B_{t} .
$$

Proof. Suppose not and let $t$ be minimal such that the claim does not hold. Thus there must exist an $x \in \mathbb{N}$ such that either

$$
x \in\left(A \cap\left\{0,1, \ldots, k_{1} k_{2} \cdots k_{t}-1\right\}\right) \triangle A_{t}
$$

or

$$
x \in\left(B \cap\left\{0,1, \ldots, k_{1} k_{2} \cdots k_{t}-1\right\}\right) \triangle B_{t}
$$

where $\triangle$ denotes the symmetric difference of sets. Pick a minimal such $x$. Let us assume that $t$ is odd and $t \geq 3$; the proof is trivial for $t=0$ or $t=1$ and similar when $t \geq 2$ is even. As $t$ is odd (and minimal), $B_{t}=$ $B_{t-1}=B \cap\left\{0,1, \ldots, k_{1} \cdots k_{t-1}-1\right\} \subset B \cap\left\{0,1, \ldots, k_{1} \cdots k_{t}-1\right\}$, thus $B_{t} \backslash\left(B \cap\left\{0,1, \ldots, k_{1} \cdots k_{t}-1\right\}\right)$ is empty.

Now write

$$
x=\sum_{i=1}^{t} k_{1} k_{2} \cdots k_{i-1} a_{i}
$$


in the mixed-radix representation with bases $\left(k_{i}\right)_{i=1}^{\infty}$. Set

$$
z=\sum_{i=0}^{\lfloor t / 2\rfloor} k_{1} \cdots k_{2 i} a_{2 i+1}, \quad w=\sum_{i=1}^{\lfloor t / 2\rfloor} k_{1} \cdots k_{2 i-1} a_{2 i} .
$$

By definition, $z \in A_{t}, w \in B_{t}=B_{t-1}$ and $x=z+w$. By the minimality of $t, B_{t-1} \subset B$, thus $w \in B$. We now distinguish the remaining three cases.

(i) Suppose $x \in\left(A \cap\left\{0,1, \ldots, k_{1} \cdots k_{t}-1\right\}\right) \backslash A_{t}$. Since $x \notin A_{t}$, we have $x \neq z$, thus $z \in A$ by minimality of $x$. Now $x, z \in A$ and $0, w \in B$. But $x+0=z+w$, contradicting the fact that $A$ and $B$ are co-Sidon.

(ii) Suppose $x \in A_{t} \backslash\left(A \cap\left\{0,1, \ldots, k_{1} \cdots k_{t}-1\right\}\right)$. As $A+B=\mathbb{N}_{0}$, we can write $x=a+b$ with $a \in A, b \in B$. Note that $x \leq k_{1} k_{2} \cdots k_{t}-1$ and this implies $x \notin A$. In particular, $x \neq a$. We claim that $x=b$. If not, then $0<a, b<x$ and the minimality of $x$ implies that $a \in A_{t}$ and $b \in B_{t}$. But $a+b=x \in A_{t}$, which contradicts the definition of $A_{t}$ and $B_{t}$. Thus we may suppose $x=b$, i.e., $x \in A_{t} \cap B$.

For $0 \leq i \leq\lfloor t / 2\rfloor-1$, define

$$
\alpha_{2 i+1}=\left\{\begin{array}{ll}
k_{2 i+1}-a_{2 i+1} & \text { if } a_{2 i+1}>0, \\
0 & \text { if } a_{2 i+1}=0,
\end{array} \quad \beta_{2 i+2}= \begin{cases}0 & \text { if } \alpha_{2 i+1}=0, \\
1 & \text { if } \alpha_{2 i+1}>0 .\end{cases}\right.
$$

Let

$$
\begin{aligned}
& u=\left(\alpha_{t-1} 0 \alpha_{t-4} \ldots \alpha_{3}-\alpha_{1}\right)_{\left(k_{i}\right)}=\sum_{i=0}^{\lfloor t / 2\rfloor-1} k_{1} \cdots k_{2 i} \alpha_{2 i+1} \in A_{t-2}, \\
& v=\left(\beta_{t-1} 0 \beta_{t-3} 0 \ldots \beta_{2} 0\right)_{\left(k_{i}\right)}=\sum_{i=1}^{\lfloor t / 2\rfloor} k_{1} \cdots k_{2 i-1} \beta_{2 i} .
\end{aligned}
$$

By definition of $k_{t}, a_{t} \prod_{i=0}^{t-1} k_{i} \in A$, and by minimality of $t$, we have $u \in A$ and $v \in B$. Clearly, $u \neq a_{t} \prod_{i=0}^{t-1} k_{i}$. But $u+x=a_{t} \prod_{i=0}^{t-1} k_{i}+v$, contradicting the fact that $A$ and $B$ are co-Sidon.

(iii) Suppose $x \in\left(B \cap\left\{0,1, \ldots, k_{1} \cdots k_{t}-1\right\}\right) \backslash B_{t}$. Clearly, $x \notin A$, otherwise $0, x \in A \cap B$, which contradicts $A, B$ being co-Sidon. Also, $x \notin A_{t}$, otherwise $x \in A_{t} \cap B$ and we can continue as at the end of case (ii). Thus $x \neq z$, and this implies $z \in A$ by the minimality of $x$. Also, $w \in B_{t}$ implies $x \neq w$. Now $0+x=z+w$, with $0, z \in A$ and $x, w \in B$, contradicting the fact that $A$ and $B$ are co-Sidon.

To complete the proof of the theorem, we must show that $k_{t} \geq 2$ for all $t>0$. Suppose that $k_{t_{0}}=1$. That is, $c_{t_{0}}=k_{1} k_{2} \cdots k_{t_{0}-1}$ is in neither $A$ nor $B$. But then, as $A$ and $B$ are perfect co-Sidon, there exist $a \in A$ and $b \in B$ such that $a+b=c_{t_{0}}$. By assumption, $a, b<c_{t_{0}}$. But clearly $(a, b) \notin$ $A_{t_{0}} \times B_{t_{0}}$ as $A_{t_{0}}+B_{t_{0}} \subset\left\{0,1, \ldots, c_{t_{0}}-1\right\}$, contradicting Claim 13. 
Theorem 12 allows us to make a useful observation about the structure of perfect co-Sidon sets.

COROLlary 14. If $A$ and $B$ are infinite perfect co-Sidon sets then for all $m \in \mathbb{N}$ there are infinitely many $n \in \mathbb{N}$ such that

$$
\{n, n+1, \ldots, 2 n+m\} \cap A=\emptyset .
$$

Proof. As the statement remains true when we translate $A$ or $B$, it suffices to prove it for $A$ and $B$ with $0 \in A \cap B$. There exists an infinite sequence of integers $\left(k_{i}\right)$ with $k_{i} \geq 2$ for all $i$ such that $A$ and $B$ are represented by the bases $k_{i}$ as in Theorem 12. Fix $m \in \mathbb{N}$ and let $t$ be such that $2 \prod_{i=0}^{t-1} k_{i}-3 \geq m$ and $\left(k_{t}-1\right) \prod_{i=0}^{t-1} k_{i} \in A$. Then by Theorem 12 the next element in $A$ is exactly $\prod_{i=0}^{t+1} k_{i}$. Let $n=\left(k_{t}-1\right) \prod_{i=0}^{t-1} k_{i}+1$. Now

$$
\begin{aligned}
\prod_{i=0}^{t+1} k_{i} & =k_{t+1}\left\{\left(k_{t}-1\right)+1\right\} \prod_{i=0}^{t-1} k_{i} \geq 2\left\{n-1+\prod_{i=0}^{t-1} k_{i}\right\} \\
& \geq 2 n-2+m+3=2 n+m+1 .
\end{aligned}
$$

Thus $\{n, n+1, \ldots, 2 n+m\} \cap A=\emptyset$. Since $A$ is infinite, it follows that for every $m$ there are infinitely many such $n$.

It is natural to ask whether all co-Sidon sets $A, B$ are subsets of perfect co-Sidon sets $A^{*}, B^{*}$. The answer turns out to be no, as the following proposition shows.

Proposition 15. The sets $A=\left\{2^{k}: k \in \mathbb{N}, k \geq 9\right\}$ and $B=\left\{3^{l}:\right.$ $l \in \mathbb{N}, l \geq 9\}$ are co-Sidon and there are no perfect co-Sidon sets $A^{*}, B^{*}$ such that $A \subseteq A^{*}$ and $B \subseteq B^{*}$.

Proof. The Diophantine equation $2^{k}+3^{l}=2^{m}+3^{n}$ with $k<m$ and $l>n$ has only five solutions (see [10]); all have exponents less than 9 . This implies that $A$ and $B$ are co-Sidon.

Note that, for all $n \geq 2^{9}, A$ contains numbers between $n$ and $2 n$. That is, for all $n, A \cap\{n, n+1, \ldots, 2 n\} \neq \emptyset$. However, if $A^{*}$ and $B^{*}$ are perfect co-Sidon sets such that $A \subset A^{*}$ and $B \subset B^{*}$, then according to Corollary 14 there is an $n$ with $A^{*} \cap\{n, n+1, \ldots, 2 n+m\}=\emptyset$.

3. Representation function. We seek to provide sufficient conditions on $A$ and $B$ so that the representation function $r(A, B, n)=\mid\{(a, b) \in$ $A \times B: a+b=n\} \mid$ is (eventually) increasing. For $C \subset \mathbb{N}_{0}$ let us denote its complement by $\bar{C}=\mathbb{N}_{0} \backslash C$.

It is easy to see that if either $A$ or $\bar{A}$ is finite and either $B$ or $\bar{B}$ is finite then $r(A, B, n)$ is eventually monotone. Indeed, if $\bar{A}$ and $B$ are finite, then for all $n>\max (\bar{A})+\max (B)$ we see that $b \in B$ implies $n-b \in A$ and thus $r(A, B, n)=|B|$. Also, if $\bar{A}$ and $\bar{B}$ are finite, then for all $n>$ 
$\max (\bar{A})+\max (\bar{B})$ we have $r(A, B, n)=n+1-|\bar{A}|-|\bar{B}|$. Finally, if $A$ and $B$ are both finite then it is obvious that $r(A, B, n)$ is eventually monotone. So the study is non-trivial only in the case when $A$ and $\bar{A}$ are both infinite.

Proposition 16. Let $A, B \subset \mathbb{N}_{0}$ be infinite perfect co-Sidon sets such that $A+B=\mathbb{N}_{0}$. Then, for any $A^{\prime} \subset A$ and $B^{\prime} \subset B$, the representation function $r\left(A+B^{\prime}, B+A^{\prime}, n\right)$ is increasing.

Proof. Note that

$$
\begin{aligned}
r\left(A+B^{\prime}, B+A^{\prime}, n\right) & =r\left(\bigcup_{b \in B^{\prime}} A+b, \bigcup_{a \in A^{\prime}} B+a, n\right) \\
& =\sum_{a \in A^{\prime}, b \in B^{\prime}} r(A+b, B+a, n) .
\end{aligned}
$$

The second equality holds because the unions are disjoint.

From $A+B=\mathbb{N}_{0}$ it follows that $(A+b)+(B+a)=\mathbb{N}_{0}+a+b$ and thus each summand is

$$
r(A+b, B+a, n)= \begin{cases}0 & \text { if } n<a+b, \\ 1 & \text { if } n \geq a+b .\end{cases}
$$

Therefore, the representation function $r\left(A+B^{\prime}, B+A^{\prime}, n\right)$ is increasing.

It follows from Theorem 12 that sets $A$ and $B$ which are infinite perfect co-Sidon exist. Since the subsets in Proposition 16 are arbitrary, we can construct many sets $A$ and $B$ such that $r(A, B, n)$ is increasing. The next theorem allows us to choose sets $A$ and $B$ whose representation function is increasing and whose counting functions $A(n)$ and $B(n)$ grow at a controlled rate.

Theorem 17. Let $A, B \subset \mathbb{N}_{0}$ be infinite perfect co-Sidon such that $A+B=\mathbb{N}_{0}$. Let $f: \mathbb{N}_{0} \rightarrow \mathbb{R}$ be such that $A(n) \leq f(n)$ and for every $M>0$ there exists $n_{0}$ such that for $n>n_{0}$ we have $f(n)<n+1-M A(n)$. Then there exists a $B^{\prime} \subseteq B$ such that

$$
\left(A+B^{\prime}\right)(n) \leq f(n) \quad \text { for all } n \in \mathbb{N}_{0}
$$

and

$$
\left(A+B^{\prime}\right)(n) \geq f(n)-A(n) \quad \text { for infinitely many } n \in \mathbb{N}_{0} .
$$

Proof. Let $A$ and $B$ be as in the statement and write $B=\left\{b_{0}<b_{1}<\cdots\right\}$. By assumption, $b_{0}=0$. Let us construct $B^{\prime} \subseteq B$ greedily as follows: set $B_{0}^{\prime}=\{0\}$ and for $i>0$ let

$$
B_{i+1}^{\prime}= \begin{cases}B_{i}^{\prime} \cup\left\{b_{i+1}\right\} & \text { if }\left(A+\left(B_{i}^{\prime} \cup\left\{b_{i+1}\right\}\right)\right)(n) \leq f_{A}(n) \text { for all } n \in \mathbb{N}_{0}, \\ B_{i}^{\prime} & \text { otherwise. }\end{cases}
$$

Then let $B^{\prime}=\bigcup_{i=0}^{\infty} B_{i}^{\prime}$. We claim that this $B^{\prime}$ satisfies the conditions of the 
theorem. By the construction,

$$
\left(A+B^{\prime}\right)(n) \leq f(n) \quad \text { for all } n \in \mathbb{N}_{0} .
$$

To prove that the other inequality holds for infinitely many values of $n$, we first need to show that $B \backslash B^{\prime}$ is infinite. Suppose that $B \backslash B^{\prime}$ is finite, and let $M=\left|B \backslash B^{\prime}\right|$. Since $A+B \backslash B^{\prime}=\bigcup_{b \in B \backslash B^{\prime}}(A+b)$ we have $\left(A+B \backslash B^{\prime}\right)(n) \leq$ $M A(n)$ for every $n$. Now, clearly,

$$
\bigcup_{b \in B^{\prime}}(A+b)=\mathbb{N}_{0} \backslash \bigcup_{b \in B \backslash B^{\prime}}(A+b) .
$$

It follows that $\left(A+B^{\prime}\right)(n)=n+1-\left(A+\left(B \backslash B^{\prime}\right)\right)(n) \geq n+1-M A(n)$ for all $n$. But, for large enough $n$, we have $n+1-M A(n)>f(n)$. Then for large enough $n$ we would have $\left(A+B^{\prime}\right)(n)>f(n)$, which contradicts the construction of $B^{\prime}$. Hence $B \backslash B^{\prime}$ is infinite.

Therefore, for infinitely many $i$, we have $b_{i+1} \notin B^{\prime}$. For such an $i$ we have $B_{i+1}^{\prime}=B_{i}^{\prime}$. Therefore, by definition of $B_{i+1}^{\prime}$, there exists $n_{i+1}$ such that $\left(A+B_{i}^{\prime} \cup\left\{b_{i+1}\right\}\right)\left(n_{i+1}\right)>f\left(n_{i+1}\right)$. Note that $n_{i+1} \geq b_{i+1}$, because for all $n<b_{i+1}$,

$$
\left(A+B_{i}^{\prime} \cup\left\{b_{i+1}\right\}\right)(n)=\left(A+B_{i}^{\prime}\right)(n) \leq f_{A}(n) .
$$

Therefore there are infinitely many $n$ such that

$$
\left(A+B^{\prime}\right)(n) \geq\left(A+B_{i}^{\prime}\right)(n) \geq f(n)-A(n) .
$$

Our main theorem follows as a corollary of Theorem 17. We restate it here for easy reference:

Theorem 3. For all $0 \leq \alpha, \beta<1,1 / 2<c_{1}, c_{2} \leq 1$, there exist sets $A, B \subset \mathbb{N}_{0}$ such that $r(A, B, n)$ is increasing in $n$, and

$$
\limsup _{n \rightarrow \infty} \frac{A(n)}{n^{c_{1}}}=\alpha, \quad \limsup _{n \rightarrow \infty} \frac{B(n)}{n^{c_{2}}}=\beta .
$$

Proof. Suppose we are given constants $0 \leq \alpha<1$ and $1 / 2<c_{1} \leq 1$. Let $A_{0}, B_{0}$ be perfect co-Sidon sets such that $A_{0}(n)=\Theta\left(n^{1 / 2}\right), B_{0}(n)=\Theta\left(n^{1 / 2}\right)$ (e.g. Construction 7). Let $f(n)=\alpha n^{c_{1}}+d$ where $d$ is a constant large enough such that $f(n) \geq A_{0}(n)$ for all $n$. Clearly, for all $m>0$ there exists an $n_{0}$ such that for $n>n_{0}, f(n)<n+1-m A_{0}(n)$. By Theorem 17, there is a $B^{\prime} \subset B_{0}$ such that $\left(A_{0}+B^{\prime}\right)(n) \leq f(n)$ for all $n$ and $\left(A_{0}+B^{\prime}\right)(n) \geq$ $f(n)-A_{0}(n)$ for infinitely many $n$. Set $A=A_{0}+B^{\prime}$. Then

$$
\alpha=\lim _{n \rightarrow \infty} \frac{f(n)}{n^{c_{1}}} \geq \limsup _{n \rightarrow \infty} \frac{A(n)}{n^{c_{1}}} \geq \lim _{n \rightarrow \infty} \frac{f(n)-A_{0}(n)}{n^{c_{1}}}=\alpha .
$$

We can construct $B$ in the same manner. By Proposition 16, the representation function $r(A, B, n)$ is increasing. 
By modifying the previous two proofs, we can restate Theorem 3 with either (or both) of the upper limits replaced with lower limits. The details are left to the interested reader. Theorem 3 gives a strong answer about the densities of sets $A$ and $B$ with monotone representation function $r(A, B, n)$.

When $c_{1}=c_{2}=1$ and $\alpha, \beta \in \mathbb{Q}$ we can restate Theorem 3 by replacing the upper limits with standard limits.

TheOREM 18. For all rational $0 \leq \alpha, \beta \leq 1$, there exist sets $A, B \subset \mathbb{N}_{0}$ such that $A$ has density $\alpha, B$ has density $\beta$ and $r(A, B, n)$ is increasing in $n$.

Proof. We construct $A$ and $B$ using mixed-radix representation to describe its elements. Write $\alpha=p_{1} / q_{1}$ and $\beta=p_{2} / q_{2}$ where $p_{i}, q_{i} \in \mathbb{N}$. Set $k_{1}=q_{1}, k_{2}=q_{2}$ and $k_{i}=2$ for all $i>2$. Let $A_{0}$ be the set of all integers that can be written in the form

$$
\sum_{i=0}^{k} k_{1} k_{2} \cdots k_{2 i} a_{2 i+1}
$$

where for each $i, 0 \leq a_{2 i+1}<k_{2 i+1}$. Similarly, let $B_{0}$ be the set of all integers that can be written in the form

$$
\sum_{i=1}^{k} k_{1} k_{2} \cdots k_{2 i-1} b_{2 i}
$$

where for each $i, 0 \leq b_{2 i}<k_{2 i}$. Note that $A_{0}$ and $B_{0}$ are perfect co-Sidon.

Let $A^{\prime}$ be the subset of $A_{0}$ consisting of all those integers whose $k_{1^{-}}$ digit (in the mixed-radix representation) lies in the set $\left\{0,1, \ldots, p_{1}-1\right\}$. As $p_{1} \leq q_{1}$ we must have $p_{1}-1 \leq k_{1}-1$. Thus $A^{\prime}$ is well-defined. Then $B=A^{\prime}+B_{0}$ is the set of all numbers whose $k_{1}$-digit lies in $\left\{0, \ldots, p_{1}-1\right\}$, that is, $B$ consists of the numbers congruent to $0,1, \ldots, p_{1}-1\left(\bmod q_{1}\right)$. The density of this set is clearly $p_{1} / q_{1}$.

Similarly, let $B^{\prime}$ be the subset of $B_{0}$ consisting of all those integers whose $k_{2}$-digit (in the mixed-radix representation) lies in the set $\left\{0,1, \ldots, p_{2}-1\right\}$. Again as $p_{2} \leq q_{2}$ we have $p_{2}-1 \leq k_{2}-1$ so $B^{\prime}$ is also well-defined. A similar argument holds when we are considering $A=A_{0}+B^{\prime}$. Here, $A$ is the set of numbers whose $k_{2}$-digit is in $\left\{0,1, \ldots, p_{2}-1\right\}$. Thus $A$ consists exactly of the numbers less than or equal to $\left(p_{2}-1\right) q_{1}\left(\bmod q_{1} q_{2}\right)$. This follows as the base of the first digit is $q_{1}$. Again, it is clear that $A$ has density $\left(p_{2} q_{1}\right) /\left(q_{1} q_{2}\right)=p_{2} / q_{2}$.

By Proposition 16, $r(A, B, n)$ is increasing.

Finally, we determine for which sets $A, B$ the representation function $r(A, B, n)$ is eventually strictly increasing. The corresponding question for a single set has been considered by Chen and Tang [2], who discuss when the functions $r, r_{1}, r_{2}$ are strictly increasing. When considering two sets and the function $r$, the problem turns out to be easy. 
Proposition 19. Let $A, B \subset \mathbb{N}_{0}$. Then the representation function $r(A, B, n)$ is eventually strictly increasing if and only if $\bar{A}$ and $\bar{B}$ are finite.

Proof. First, let us assume that $r(A, B, n)$ is eventually strictly increasing. We will use the trivial identity

$$
n+1=r\left(\mathbb{N}_{0}, \mathbb{N}_{0}, n\right)=r(A, B, n)+r(\bar{A}, B, n)+r(A, \bar{B}, n)+r(\bar{A}, \bar{B}, n),
$$

which is equivalent to

$$
n+1-r(A, B, n)=r(\bar{A}, B, n)+r(A, \bar{B}, n)+r(\bar{A}, \bar{B}, n) .
$$

In the last identity the left hand side is bounded, since we have assumed that $r(A, B, n)$ is eventually strictly increasing. Thus the right hand side is also bounded. Hence $r(\bar{A}, B, n), r(A, \bar{B}, n)$ and $r(\bar{A}, \bar{B}, n)$ are all bounded. From this it follows that $r\left(\bar{A}, \mathbb{N}_{0}, n\right)=r(\bar{A}, B, n)+r(\bar{A}, \bar{B}, n)$ and $r\left(\mathbb{N}_{0}, \bar{B}, n\right)=$ $r(A, \bar{B}, n)+r(\bar{A}, \bar{B}, n)$ are bounded. Thus $\bar{A}$ and $\bar{B}$ must be finite.

Now we assume that $\bar{A}$ and $\bar{B}$ are finite. For any $n>\max (\bar{A})+\max (\bar{B})$ we know that $a \in \bar{A}$ implies $n-a \notin \bar{B}$ and vice versa, so we can write

$$
r(A, B, n)=n+1-|\bar{A}|-|\bar{B}|<n+2-|\bar{A}|-|\bar{B}|=r(A, B, n+1) .
$$

Thus for $n>\max (\bar{A})+\max (\bar{B})$ the representation function is strictly increasing.

4. Open problems. A far-reaching goal would be to completely characterize co-Sidon sets. Which co-Sidon sets are subsets of some perfect co-Sidon sets? Are two random sets likely to be co-Sidon?

Can we completely characterize sets $A, B$ whose representation function is increasing? Are there constructions that do not come from perfect coSidon sets?

Acknowledgments. The authors would like to thank András Sárközy for introducing these problems and for many useful discussions.

This work was done while the third and fourth authors were guests of the Department of Mathematical Sciences at the University of Memphis.

\section{References}

[1] R. Balasubramanian, A note on a result of Erdös, Sárközy and Sós, Acta Arith. 49 (1987), 45-53.

[2] Y. Chen and M. Tang, On the monotonicity properties of additive representation functions, II, preprint.

[3] P. Erdős, A. Sárközy and V. T. Sós, Problems and results on additive properties of general sequences, III, Studia Sci. Math. Hungar. 22 (1987), 53-63; IV, in: Number Theory, K. Alladi (ed.), Lecture Notes in Math. 1122, Springer, Berlin, 1985, 85-104; $V$, Monatsh. Math. 102 (1986), 183-197. 
[4] P. Erdős and P. Turán, On a problem of Sidon in additive number theory, and on some related problems, J. London Math. Soc. 16 (1941), 212-215.

[5] H. Halberstam and K. F. Roth, Sequences, Vol. 1, Oxford Univ. Press, 1966.

[6] F. Krückeberg, $B_{2}$-Folgen und verwandte Zahlenfolgen, J. Reine Angew. Math. 206 (1961), 53-60.

[7] I. Z. Ruzsa, Solving a linear equation in a set of integers I, Acta Arith. 65 (1993), 259-282.

[8] A. Sárközy, Unsolved problems in number theory, Period. Math. Hungar. 42 (2001), $17-35$.

[9] A. Stöhr, Gelöste und ungelöste Fragen über Basen der natürlichen Zahlenreihe II, J. Reine Angew. Math. 194 (1955), 111-140.

[10] R. Tijdeman and L. Wang, Sums of products of powers of given prime numbers, Pacific J. Math. 132 (1988), 177-193; Corrigendum, ibid. 135 (1988), 396-398.

Department of Mathematical Sciences

University of Memphis

Memphis, TN 38152-3240, U.S.A.

E-mail: fabriciosb@gmail.com

hulgan@yahoo.com

ago.riet@gmail.com

Alfréd Rényi Institute of Mathematics

P.O. Box 127

H-1364 Budapest, Hungary

E-mail: corypalmer@gmail.com
Central European University

Frankel Leó út 30-34

H-1023 Budapest, Hungary

E-mail: nlemons@gmail.com

Department of Mathematics

School of Arts and Sciences

University of Pittsburgh

301 Thackeray Hall

Pittsburgh, PA 15260, U.S.A.

E-mail: jpwheelr@memphis.edu

Received on 30.12.2008

and in revised form on 10.3.2009 